\title{
Protocol for Implementation of an Evidence Based Parentally Administered Intervention for Preterm Infants
}

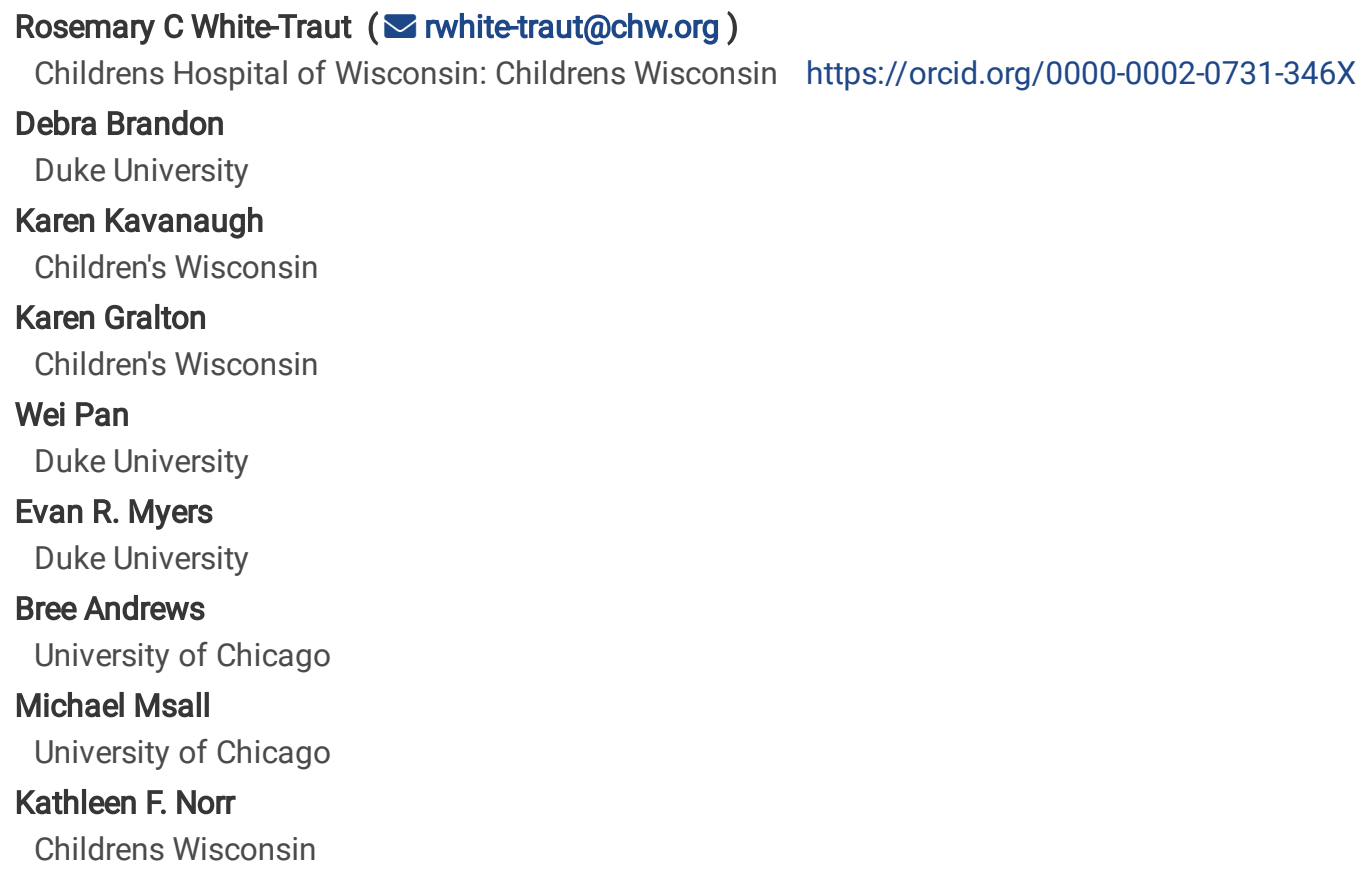

Research article

Keywords: Preterm infant, Behavioral Intervention, Parent Engagement, NICU Implementation, CFIR

Posted Date: December 31st, 2020

DOI: https://doi.org/10.21203/rs.3.rs-136236/v1

License: (c) (i) This work is licensed under a Creative Commons Attribution 4.0 International License. Read Full License 


\section{Abstract}

\section{Background}

Multi-sensory behavioral interventions for preterm infants have the potential to accelerate feeding, growth, and optimize developmental trajectories and increase parents' interactive engagement with their infants. However, few neonatal intensive care units (NICUs) provide evidence-based standardized early behavioral interventions as routine care. Lack of implementation is a major gap between research and clinical practice. H-HOPE, is a standardized behavioral intervention with an infant- directed component (Massage+) and a parent-directed component (four participatory guidance sessions that focus on preterm infants' behaviors and appropriate responses). H-HOPE has well documented efficacy. The purpose of this implementation study is to establish H-HOPE as the standard of care in 5 NICUs.

Methods

The study employs a Type 3 Hybrid design to simultaneously examine the implementation process and effectiveness in five NICUs. To stagger implementation across the clinical sites, we use an incomplete stepped wedge design. The five participating NICUs were purposively selected to represent different acuity levels, number of beds, locations and populations served. Our implementation strategy integrates our experience conducting H-HOPE and a well-established implementation model, the Consolidated Framework for Implementation Research (CFIR). The CFIR identifies influences (facilitators and barriers) that affect successful implementation within five domains: intervention characteristics, outer setting (the hospital and external events and stakeholders), inner setting (NICU), implementers' individual characteristics, and the implementation process. NICUs will use the CFIR process, which includes three phases: Planning and Engaging, Executing, and Reflecting and Evaluating. Because sustaining is a critical goal of implementation, we modify the CFIR implementation process by adding a final phase of Sustaining.

\section{Discussion}

This study builds on the CFIR, adding Sustaining H-HOPE to observe what happens when sites begin to maintain implementation without outside support, and extends its use to the NICU acute care setting. Our mixed methods analysis systematically identifies key facilitators and barriers of implementation success and effectiveness across the five domains of the CFIR. Long term benefits have not yet been studied but may include substantial health and developmental outcome for infants, more optimal parent-child relationships, reduced stress and costs for families, and substantial indirect societal benefits including reduced health care and special education costs.

Trial registration

ClinicalTrials.gov registration number NCT04555590, Registered on 8/19/2020

\section{Background}

The high cost of preterm birth. Premature birth is associated with immaturity of major organs and regulatory behaviors, feeding difficulties, and slower early growth, leading to longer initial hospitalization and increased economic costs [1-14]. Parents of preterm infants also experience an increase in stress, anxiety, depressive symptoms, and lack of confidence about parenting, which also leads to less optimal parent-infant interaction and adversely impacts infant growth and development [15-25]. Preterm births account for only $10-12 \%$ of births in the US, but the cost of preterm birth accounts for more than $40 \%$ of the total costs of birth hospitalizations $[1,5,7,26,27]$. Post-discharge, $19 \%$ of preterm infants experience ongoing medical conditions, increasing average outpatient costs in the first year to approximately $\$ 10,000$, and prescription costs to approximately $\$ 1,800[27,28]$. The first year total health care costs for preterm infants are three times higher when compared to full-term infants, reaching \$14 billion in 2017 [29]. For very preterm infant survivors (28-31 weeks gestation), and extremely preterm infant survivors ( $<28$ weeks gestation), these medical costs are even higher.

Early developmental interventions have the potential to improve outcomes for preterm infants and families. Importantly, early investment in preterm infant development has high potential impact on infant short and long term outcomes, parent-infant relationships and public health and education costs [5, 7, 27, 30]. The Physical Environment Exploratory Group endorses use of sensory interventions, including massage, as standard care after 30-31 weeks post-menstrual age (PMA, calculated as gestational age at birth plus chronological age) [31]. A large body of evidence supports that these interventions accelerate early brain development and maturation of visual function, improve feeding ability and growth factors, as well as health, long-term language, motor, and cognitive development. For the parents, the interventions increase interactive engagement with their infants and parent-infant interaction, which may mitigate the effects of increased parental stress [18, 31-47]. Multi-sensory developmental interventions that have a behavioral focus have been found especially effective in accelerating feeding, growth, and development [31, 32, 42, 48, 49]. However, few neonatal intensive care units (NICUs) provide evidence- 
based standardized parent directed early developmental interventions that have a behavioral focus as routine care. Lack of implementation is a major gap between research and clinical practice.

$\mathrm{H}-\mathrm{HOPE}$ is a standardized intervention ready for dissemination. Our team developed the H-HOPE intervention (Hospital to Home: Optimizing the Preterm Infant's Environment) to promote early infant development and parental engagement. H-HOPE consists of two components, one infant-directed and one parent-directed. Together, these components simultaneously address the needs of both preterm infants and their parents, especially their need for mutual engagement in NICUs. Our early research documented the efficacy of H-HOPE's infant-directed component, the ATVV, which provides Auditory (voice), Tactile (moderate touch massage), Visual (eye to eye), and Vestibular (rocking) stimulation. We renamed the ATVV as Massage+ to be more readily understood by parents. Massage+ is initiated when infants are ready for social interaction around 31-32 weeks. When tested with infants at varying degrees of risk, Massage+ improved infant oral feeding and social interactive skills prior to and during feeding, improved their capacity to attain alert behavioral states more optimal for feeding and parent-infant interaction, in-hospital growth (weight gain) and development, and reduced length of hospital stay [11-14, 50-54]. However, Massage+ alone did not address parents' reported distress and their need for participatory guidance and social support to engage with their infants, despite the infants' immature behaviors. To meet parents' needs, we developed the parent-directed component of H-HOPE, which consists of 4 participatory guidance sessions for parents, 2 during the NICU stay and 2 during the transition to home.

Together, H-HOPE's components optimize early infant behavior and parental capacity to engage in social interaction. In previous research, we found improved developmental maturation (more mature behavioral states, increased frequency of orally directed behaviors, faster transition from gavage to oral feeding, improved sucking organization and motor development), greater in-hospital growth (weight gain and length), and enhanced engagement, social interaction and infant responsivity $[42,48,49,55]$. Additionally, $\mathrm{H}$-HOPE reduced initial inhospital costs (net savings of $\$ 13,976$ per infant after adjusting for the mean intervention cost of $\$ 680$ ) [56] and health care use from discharge through 6-weeks corrected age (CA, chronological age corrected for weeks born preterm) [57]. With established efficacy and a manualized standardized protocol, $\mathrm{H}$-HOPE is ready for widespread implementation, making $\mathrm{H}$-HOPE uniquely suited as an early behavioral intervention for preterm infants in NICUs.

\section{Preparing NICUs to adapt H-HOPE as the standard of care}

To reduce the gap between research and implementation, clinicians need a strategy to introduce an early behavioral intervention involving parents within the high-acuity NICU. Our implementation strategy integrates our experience conducting $\mathrm{H}$-HOPE and a well-established implementation model, the Consolidated Framework for Implementation Research (CFIR) [58]. The CFIR identifies influences (facilitators and barriers) that affect successful implementation within five domains: intervention characteristics, outer setting (the hospital and external events and stakeholders), inner setting (NICU), implementers' individual characteristics, and the implementation process. We selected the CFIR because the framework integrates the best features of previous models [58] and captures the complexity of real-world implementation to identify site-specific factors and patterns across cases $[59,60]$. The framework has been used for diverse interventions in inpatient, clinic and community settings. A systematic review in 2016 identified 26 empirical studies using the CFIR, [61] and there are numerous studies published since then [42, 62-70]. However, only two previous studies occurred in a high acuity inpatient setting (adult acute care units) $[62,69]$ and no prior studies include the NICU setting. The CFIR provides a systematic approach that will be used to guide the process of implementing H-HOPE in NICUs, to recognize facilitators of successful implementation, and to identify and address implementation barriers across different sites. NICUs will use the CFIR process, which includes three phases: Planning and Engaging, Executing, and Reflecting and Evaluating. We modified the CFIR implementation process by added a final phase of sustaining H-HOPE without continued support from the research team, because sustaining is a critical goal of implementation [71, 72].

In addition to adopting an implementation model, we modified the H-HOPE protocol used in the efficacy study to further facilitate implementation of H-HOPE as the standard of care for this initial study, nationwide and eventually globally. We expanded infant and parent eligibility criteria to reach a wider range of preterm infants and their parents who can benefit from an early behavioral intervention. In collaboration with Pathways.org, an organization dedicated to early childhood development, we developed a web-based support package of training webinars, Massage+ videos and downloadable materials for H-HOPE providers and trainers (www.Pathways.org). We replaced costly post-discharge home visits with screen time (e.g. FaceTime). Through regular interactive consultation at each site, the research team will share their experiences to support implementation and lessons learned across sites will be incorporated into the web-based support package. Because every NICU represents a different context, we view the implementation process as a collaboration that balances fidelity to intervention core components to maintain H-HOPE's effectiveness, while adapting non-essential aspects (e.g. training format, who provides services) to fit each site's unique set of needs $[73,74]$. 


\section{Study Aims}

\section{Aim 1 (Implementation Success)}

Identify the degree of implementation success at each clinical site every 2 months from Executing through 6 months after supported implementation ends (primary implementation outcome). Success measures include: Sustainability (offering H-HOPE), Reach (\% of eligible parent-infant units receiving H-HOPE) and Degree of Implementation (mean H-HOPE services received).

\section{Aim 2 (Effectiveness)}

Evaluate the effectiveness of H-HOPE compared to a pre-H-HOPE comparison cohort. We hypothesize that the H-HOPE cohort will have:

H2a. More positive infant outcomes including improved growth in the NICU and fewer illness visits from initial discharge through 6-weeks post discharge (primary H-HOPE effectiveness outcomes).

$\mathrm{H} 2 \mathrm{~b}$. Lower initial hospitalization costs from entry into H-HOPE through discharge, taking into account the implementation costs of the $\mathrm{H}-$ HOPE program per infant, resulting in long-term hospital/health system cost savings (secondary H-HOPE effectiveness outcome).

$\mathrm{H} 2 \mathrm{c}$. More positive parent outcomes, including increased visits in the NICU, lower NICU- related worry, lower anxiety and depression, and greater confidence in premature infant care (exploratory H-HOPE effectiveness outcomes).

\section{Aim 3 (Influences associated with success and effectiveness)}

Determine the influences of the 5 CFIR domains associated with success (Aim 1) and the primaryH-HOPE effectiveness infant outcomes (Aim 2).

\section{Design}

The study employs a Type 3 Hybrid design [75] to simultaneously examine the implementation process and effectiveness in five NICUs. To stagger implementation across the clinical sites, we use an incomplete stepped wedge design [76] (see Figure 1). This design is more advantageous than conventional cluster randomized trials because it requires fewer NICUs (clusters), allows all NICUs to implement $\mathrm{H}$ HOPE, and permits an estimate of treatment effects both within- and between-NICUs [76-79]. To eliminate systematic bias due to order of initiation, start dates were determined by a random number generator. Each NICU implements H-HOPE in 3 phases: Planning and Engaging (6 months), Executing (6 months), and Reflecting and Evaluating (2 months) [80, 81]. Implementation is followed by 6 months of Sustaining.

To evaluate effectiveness, we chose not to use a randomized design with simultaneous intervention and control comparison groups because it would be confusing for nursery staff and parents to have two different standards of care. Because this is an implementation study, H-HOPE will be introduced as the standard of care. All eligible infants will receive H-HOPE whether or not their parents enroll in the research study. Instead, we compare parent and infant outcomes before and after the introduction of H-HOPE at each site.

\section{Study Setting And Sample}

To maximize generalizability of results, we selected 5 NICUs that are diverse in size (26-72 beds), acuity level (one level II, two level III, and two level IV NICUs), location (urban/suburban/rural), and population served. (See Table 1.) Our study population is anticipated to include 44\% White, 26.6\% Black/African American, 0.6\% Native American, 6\% Asian, 19\% Latinx, and 3.7\% other. These estimates include substantially fewer Latinx, a group with the lowest prematurity rate of $9.9 \%$, and larger proportion of Blacks and Native Americans, the groups with the highest prematurity rates in the US (Blacks, 13.8\%; Native Americans, 11.4\%) [82]. All of the NICUs provide developmental care that includes non-disruption of sleep cycles and skin-to-skin care when infants are stable. Comparisons across units will help identify $\mathrm{NICU}$ characteristics that make it more challenging to implement and sustain $\mathrm{H}$-Hope. Sites 2 and 3 are in the same health system. Therefore, these NICUs will implement H-HOPE simultaneously and are considered as one site for effectiveness evaluation (Aim 2). However, they will be analyzed as separate sites for success and influences (Aims 1 and 3 ) because the inner settings differ, e.g., they have different nursing staff and hospital administrators.

\section{Sample}

Aim 1 (Implementation success) data will be obtained from each site's de-identified aggregated electronic health record (EHR) data. 
Aim 2 (Effectiveness) participants include both parents and infants from two cohorts of subjects: the Pre-H-HOPE Comparison Cohort and the H-HOPE Cohort. The Pre-H-HOPE Comparison Cohort consists of two sub-cohorts: 1) Consented Pre-H-HOPE Parent-Infant Units, for whom all parent and infant data are collected and 2) Non-Consented Pre-H-HOPE Infants for whom only de-identified existing infant growth and cost data are used. The non-consented Pre-HOPE Cohort is added to have a large enough sample for propensity score matching to test H-HOPE's impact on infant growth and initial hospitalization costs.

Eligibility Criteria and Recruitment: For H-HOPE and Pre-H-HOPE Consented parent-infant dyads, infants are eligible if they are born at 2335 weeks gestational age, at least 31-32 weeks PMA, and clinically stable. Parents are mothers, fathers, or other significant family member who intend to act in the role of a parent, regardless of biological relationship to the infant, gender identity or sexual orientation. In most cases the biological mother is identified as the primary parent and she will be asked to name the second parent. The Non-Consented Pre-H-HOPE Infants must meet the same infant criteria, however no parents are involved.

\section{Sample size and power for Aim 2 Effectiveness}

Based on participating sites' data, 542 infants will be eligible for the H-HOPE cohort during the 6-month Executing phase. Of the available infants, we expect to enroll at least 70\% ( $n=379$ parent-infant pairs) and to retain 265 through 6-weeks following the infant's discharge. During a 12-month period before H-HOPE implementation, 1084 eligible infants will be available for the Pre-H-HOPE Comparison Cohort in two sub-cohorts.

The first sub-cohort, the Consented Pre-H-HOPE Parent-Infant Units, is recruited at the same time as the Planning and Engaging phase at each site and will contribute to all outcome analyses $(n=542)$. The retention rates will be the same in the Consented Pre-H-HOPE subcohort and the H-HOPE cohort $(n=265)$. The second sub-cohort, the Non-Consented Pre-H-HOPE Infants $(n=542)$, will contribute only retrospective infant growth and cost data obtained from de-identified EHR and cost records in the 6 months prior to the start of Planning and Engaging. Based on our prior research, near medium to large effect sizes are expected for the primary outcomes of infant growth and

health care utilization following discharge $[48,56]$ and the secondary hospital cost outcome [57]. Using the STATA package stepped wedge [83], the power analysis found that there will be at least $80 \%$ of power to detect a medium effect of Cohen's $d=-0.50$ at a significance level of $a=.05$ in this stepped wedge design with 4 sites and an average of at least 50 subjects per site (or 200 in total) across 4 staggered time points, assuming the intra-class correlation coefficient (ICC) is .1, a common ICC found in health and behavioral research [84, 85]. Assuming ICC as .05, which is common ICC health cost analysis [86-88], research would require at least 30 subjects per site (or 120 in total). Thus, in this proposed study, the anticipated total sample size of 265 will be adequate to detect a medium effect for all outcome measures.

The Aim 3 sample includes hospital personnel and parents receiving H-HOPE at each site. Consented H-HOPE parents will be randomly selected ( 15 parents per site, 75 total) to be interviewed prior to discharge and when their infant is 6-weeks post discharge. Purposively selected hospital personnel include at least one hospital administrator, NICU leadership (e.g. medical director and nurse manager), clinical site H-HOPE team members (2-4 per site), and selected staff who work in the NICU (10 per site) who have delivered H-HOPE, especially champions and non-supporters. Each of these selected hospital personnel will participate in 3 short qualitative interviews about their experience with H-HOPE. In addition, all registered staff nurses who work at least $50 \%$ in the NICU will complete a brief survey regarding engagement and burnout during the three project phases.

\section{Implementation Study Conditions}

Infants and parents in the Pre-H-HOPE Comparison Cohort receive usual NICU care and parent interactions with NICU staff.

The H-HOPE Cohort of infants and parents receive H-HOPE in-hospital and during the transition to home. Four parent-directed participatory guidance sessions offer social support to parents and help them learn the nuances of preterm infant behavior and how to interact with their infant and provide Massage+. The initial in-hospital session occurs when H-HOPE begins. Preparing for the transition to home begins during the second in-hospital session shortly before discharge, and continues with sessions at 2-3 days and 7-15 days post-discharge via screen time. The infant-directed multi-sensory component of H-HOPE Massage+, is administered for 15 minutes prior to a feeding twice daily, starting when the infant is physiologically stable and ready for social interaction ( $\approx 31-32$ weeks PMA for infants $<30$ GA at birth). Twice daily administration was selected based on our previous research [11, 12, 14, 42, 49, 51-53, 55, 89-91]. Ideally, parents would always administer Massage+, however few parents are able to visit twice daily. Therefore, trained staff will provide the intervention in the parents' absence. Parents will continue the Massage+ at home twice daily until 1-month CA. In our previous research with low income minority parents, $95 \%$ continued Massage+ at home $[42,51]$. 
To assess H-HOPE fidelity during Executing at each site, we document completeness and quality of services provided for each parentinfant unit, as recommended in recent studies [92, 93]. Completeness of services in-hospital and during the transition to home is recorded in the EHR and assessed as part of Aim 1. The quality of H-HOPE services is examined periodically using standardized forms by site staff to ensure that fidelity at that site is maintained over time.

The H-HOPE Implementation Support Package consists of 2 components. A multimedia web-based program provides materials and training which are available to parents and staff on the Pathways.org website (www.Pathways.org). Interactive assistance to each site provides NICU-specific consultation throughout the 3 phases of implementing H-HOPE. This consultation has been shown to facilitate implementation $[81,94]$ On line webinars will be supplemented by in person training and support at the infant's bedside, especially when the staff administer H-HOPE for the first time. Each site will have several master trainers. A program for master trainers will be developed using examples from the first two sites trainings.

\section{Measures.}

Aim 1 primary implementation outcome measures. We examine three measures of success recommended for late-phase implementation by Proctor et al [71]. Sustainability measures whether the site is providing H-HOPE. Reach measures the proportion of eligible parent-infant units who receive H-HOPE at that site. Degree of Implementation measures the mean H-HOPE services received per parent-infant unit. The research team will consult with each site to build within the EHR infant eligibility for H-HOPE, documentation of Massage+ and the parent participatory guidance sessions. These success measures will be obtained from each site's EHR which will record eligibility and receipt of H-HOPE parent and infant services. If the site chooses to discontinue H-HOPE, we will conduct the final interview and end evaluation.

Aim 2 effectiveness outcome measures. Our primary effectiveness outcome measures include infant growth from birth through hospital discharge and acute care visits from initial discharge through 6-weeks post discharge. Our secondary outcome is mean hospitalization cost per day from entry into H-HOPE until discharge adjusted for the per-infant cost of providing H-HOPE. Our exploratory outcome includes 3 parent measures (See Table 2).

Covariates for Aim 2 analyses include infant sex, partial implementation of H-HOPE, and number of parent NICU visits. Other Infant and parent characteristics will be included as covariates if they are related to the specific outcome variable of interest. Infant characteristics include race/ethnicity, gestational age at birth, single/multiple birth, sex, Neurobiological Risk Score (NBRS) [95, 96], and days of mechanical ventilation. Parent characteristics include primary parent education, age, income, marital status, self-identified race/ethnicity, relationship to the infant, and maternal parity.

Aim 3 Secondary Implementation Outcome Measures. Aim 3 uses mixed methods analyses to integrate data collected for this aim regarding influences (facilitators or barriers) in each of the 5 CFIR domains with data from Aim 1 (implementation success) and Aim 2 (effectiveness for the primary infant outcomes). Data specific for Aim 3 include: qualitative interviews with hospital personnel and parents, H-HOPE team meeting records, research team observation notes, nurse survey data, and fidelity data from H-HOPE records. Separate interview guides for hospital personnel and parents include questions about domains and constructs within the CFIR framework relevant to their experience with H-HOPE. Hospital personnel are interviewed at 3 time points (Planning and Engaging, end of Executing, and end of Sustaining). Data about individual characteristics that influence implementation come from registered staff nurse surveys conducted at the same time as the interviews. Parents are interviewed twice: close to discharge and again after discharge when the infant has been home for 6 weeks.

\section{Study Procedures}

Overall study coordination. Procedures for this multisite complex implementation and evaluation study, are coordinated within three Centers: the Implementation Center, the Quantitative Data Center, and the Qualitative and Mixed Methods Data Center. Each Center is guided by a co-investigator who works collaboratively to facilitate overall project coordination and management.

The Implementation Center coordinates the implementation of H-HOPE at each clinical site, working closely with the H-HOPE Clinical Site Implementation Team. Each site's H-HOPE team is comprised of the Clinical Site Nurse, NICU leadership and other clinicians.

Collaboratively with the co-investigator and the study's Project Director, the site H-HOPE team develops the site-specific NICU protocol and plan for staff training, coordinates the implementation process in the $\mathrm{NICU}$, and serves as champions who provide resources and support for staff throughout implementation.

During the Planning and Engaging phase, the Implementation Center will train the clinical site's H-HOPE Implementation team. The H-HOPE Team in collaboration with the Center Director, will identify the necessary processes for establishing the H-HOPE program as standard of 
care, taking into account the NICU culture and their capability for adaptation. During the Executing phase, the NICU will implement H-HOPE according to their plan. During the Reflecting and Evaluating phase, the H-HOPE Implementation team will participate in preparing a report describing the effectiveness of the H-HOPE program on the infants and parents in their NICU, as well as hospital personnel. This report will be shared with hospital administrators and NICU leadership to provide evidence for sustaining or ending the H-HOPE program. During independent Sustaining, the H-HOPE Implementation Team can contact the Implementation Center at any time for additional consultation.

The Quantitative Data Center provides oversight and coordination for all quantitative data-related operations and conducts the quantitative data analyses for Aim 1 (degree of implementation) and Aim 2 (effectiveness). Prior to Planning and Engaging, the Center works with each site's informatics team to modify their EHR build so the H-HOPE eligibility, initiation and specific services received can be documented directly in the EHR. The Center also oversees the REDCap database construction and management for participant screening, study enrollment, eConsenting, study monitoring, and quantitative data collection. During implementation at each site, the Center works closely with each site to obtain relevant data throughout the study, shares site results and helps prepare the report that the site administrative team will use to decide whether to continue H-HOPE as the standard of care. Each site has an H-HOPE research team that is responsible for consenting both pre-H-HOPE and H-HOPE parents, facilitating and monitoring data collection, and coordinating data collection and transfer with the data Centers.

The Qualitative and Mixed Methods Data Center manages and analyzes all qualitative and mixed methods data-related operations. The primary focus of the Center is ongoing mixed methods data analysis and interpretation to address Aim 3. The qualitative interview data are managed within the Center and analyzed as described below. The relevant quantitative data are obtained from the Quantitative Data Center. At each site, the Center works with the H-HOPE team to obtain H-HOPE team meeting records and to complete interviews with selected hospital personnel and selected H-Hope parents.

\section{Analysis Plan}

Aim 1 Analysis: Aim 1 measures, Sustainability, Reach, and Degree of Implementation, are analyzed by the Quantitative Data Center, using descriptive statistics and visual displays.

Aim 2 Analysis: Aim 2 evaluates the effectiveness of H-HOPE including infant growth, health care utilization following discharge, infant hospital costs. Aim 2 also explores the impact of H-HOPE on parent outcomes detailed in Table 2 (exploratory outcomes). The Quantitative Data Center analyzes Aim 2 measures.

Before analytic modeling, propensity score methods [97-100] will be utilized to reduce any selection bias by ensuring balanced distributions of infant and primary parent characteristics between Pre-H-HOPE and H-HOPE infants. For the growth and infant hospital costs outcomes, propensity scores will first be computed using available infant and parent characteristics. Then, pairs of infants will be created by matching each infant in the H-HOPE cohort with an infant in the pre-H-HOPE cohort with the same or most similar propensity scores. The final analytical models will be applied directly to propensity-score-matched pairs.

For the other Aim 2 outcomes, including health care utilization from discharge through 6 weeks post discharge and the exploratory parent outcomes, data are collected directly from parents and are not available in hospital records. Therefore, propensity score weighting will be used. In propensity score weighting, propensity scores will first be computed similarly to propensity score matching. Then propensity scores will be used to calculate inverse probability of treatment weights as weighting scores, rather than matching samples (i.e., infants or parents). The propensity weighting scores will be incorporated in weighted final analytical models preserving the entire sample for final analytical modeling.

In all the final analytical modeling, linear mixed models will be utilized to analyze normally distributed outcomes $[79,101]$ (e.g., infant growth and parent outcomes). Linear mixed models are an extension of linear regression models to allow estimating both between-site and within-site effects when there is a nested data structure (e.g., individual patients/infants are nested within sites). Generalized linear mixed models, an extension of linear mixed models, will be used to analyze non-normally distributed outcome measures (e.g., infant health care utilization, hospital costs).

In order for new hospitals or health systems to make informed decisions about implementation, we will estimate the incremental costs of implementing H-HOPE using a Markov state-transition model over a 5-year time horizon from the perspective of an individual hospital or health system, using standard methods $[56,102,103]$. Total implementation costs will be derived from (1) H-HOPE initial implementation costs using the observed number of hours spent for training multiplied by national estimates of hourly NICU nursing salary, (2) H-HOPE infant intervention delivery costs estimated from the propensity-weighted hospitalization costs, and (3) costs of infant healthcare

Page $7 / 16$ 
utilization (number of visits multiplied by the average cost of each type of visit). In subsequent years, the costs of ongoing training will be reduced based on estimates of the number of new staff needing training. Both probabilistic and deterministic analyses will address the effect of uncertainty in cost and effectiveness estimates on the decision to implement H-HOPE, including (a) identifying the plausible values for effectiveness where H-HOPE does not result in net cost-savings over a 5 year time horizon, and (b) if cost-savings are reached within 5 years, the time for achieving cost-neutrality. Additional sensitivity analyses will examine the effects of varying assumptions about NICU admission volume and staff turnover.

Aim 3 Analyses: Aim 3 analysis will determine influences (facilitators and barriers) in each of the 5 CFIR domains that are associated with success (Aim 1) and H-HOPE effectiveness for our primary infant outcomes (Aim 2). We follow a series of tasks and create within and across case matrices to integrate qualitative and quantitative data using well-established mixed methods analyses [104] to code the key influences on implementation success from the qualitative data to create a narrative summary and within-site matrix for each site across time. Next we examine the quantitative measures from the nurse survey and H-HOPE fidelity data. These data will be pulled into the mixed methods data analysis software, where summary statistics such as mean scores can be calculated and categorized as interval level measures (e.g. high/medium/low), and then added to the case summary. Next we identify those influences linked with success and effectiveness for each site and across all sites.

We then create interval level summary categories based on the quantitative data from Aim 1 and Aim 2 and add the levels of success and effectiveness to each site's case summary and within site matrix. We combine these partial pictures into a single within-case summary matrix for each NICU. Finally, we determine common influences across sites associated with both success and effectiveness. We will display our findings in a single across-site matrix showing influences associated with implementation success and degree of effectiveness as well as factors affecting only one of these.

\section{Impact of the Covid-19 pandemic on this study}

Since this implementation research was funded, the COVID-19 pandemic has drastically altered staff and parent interactions with infants in the NICU. Currently, most sites require both staff and parents to wear masks and parent visiting has been limited. These restrictions may directly impact the effectiveness of H-HOPE as Massage+ emphasizes face-to-face contact between the infant and parent or other caregiver. We plan to document specific COVID-19 related changes at each NICU during the course of this study, and we are exploring strategies such as transparent masks to reduce the disruption of COVID-19 restrictions. We will also incorporate the impact of masks and visitation restrictions on parent-infant interactions during $\mathrm{H}-\mathrm{HOPE}$ into our analyses.

\section{Study Status.}

At the time this manuscript was submitted for publication, the research was in the initial start-up phase. The implementation process at specific sites has not yet started and no subjects have been enrolled.

\section{Discussion}

This study builds on one of the most widely used and systematic implementation frameworks, the CFIR, and extends its use to the NICU acute care setting. We adapted the 3-phase CFIR implementation model by adding Sustaining to explicitly observe what happens when sites begin to maintain implementation without outside support. Our mixed methods analysis systematically identifies key facilitators and barriers of implementation success and effectiveness across the five domains of the CFIR. The five participating NICUs were purposively selected to represent different acuity levels, number of beds, locations and populations served. This comprehensive approach allows us to examine both the final level of implementation success and different patterns by site over time.

Results of our analysis will identify common and site-specific implementation facilitators and barriers as well as ways sites overcame barriers. Findings will be widely disseminated to guide future expansion of H-HOPE as the standard of care. Study results will make major contributions to implementation science, especially for the introduction of complex evidence-based programs into the challenging highacuity environment of the NICU. If the five sites in this study are successful in sustaining the program, H-HOPE will be ready for widespread adoption nationwide.

Early behavioral interventions involving parents can engage families and improve outcomes for preterm infants and their families. Although now recommended as the standard of care, this type of early intervention is rarely provided outside of research. $\mathrm{H}$-HOPE brings a unique approach to early behavioral intervention by simultaneously addressing the needs of infant and parents. $\mathrm{H}-\mathrm{HOPE}$ is the only evidence-based and standardized early behavioral intervention that is manualized and ready for widespread adoption. $\mathrm{H}$-HOPE has already been shown to support early infant growth and development and parent-infant engagement. Long term benefits have not yet been studied 
yet may include short term health and developmental outcome for infants, more optimal parent-child relationships, reduced stress and costs for families, and substantial indirect societal benefits including reduced health care costs and need for special education services. This implementation study will provide a systematic strategy to scale-up H-HOPE as the standard of care in the complex high acuity NICU setting. If the 5 NICUs in this study succeed in implementing and sustaining H-HOPE, what is learned from this study will support other NICUs to adopt it. Our team will publish standardized recommendations for generalizability at the conclusion of this study.

\section{Abbreviations}

$\mathrm{NICU}=$ neonatal intensive care unit

H-HOPE = Hospital to Home: Optimizing the Preterm Infant's Environment

CFIR = Consolidated Framework for Implementation Research

PMA = post-menstrual age

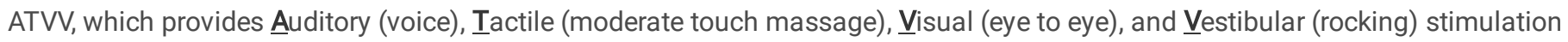

$\mathrm{CA}=$ corrected age (chronological age corrected for weeks born preterm)

$\mathrm{EHR}=$ electronic health record

ICC = intra-class correlation coefficient

NBRS = Neurobiological Risk Score

\section{Declarations}

Ethics Approval and Consent to Participate: IRB approval - PRO00035600, Medical College of Wisconsin - Title - Implementation of an Evidence Based Parentally Administered Intervention for Preterm Infants.

Consent for Publication: Not applicable.

Availability of Data and Materials: Data from this human subjects research will be shared with other investigators in accordance with the guidelines set forth by the NIH Data Sharing Policy and Implementation Guidance (March 5, 2003).

Competing Interests: There are no competing interests.

Funding: 1R01HD098095-01A1, National Institute of Child Health and Human Development, National Institutes of Health and Pathways.org

Authors' contribution: All authors read and approved the final manuscript and contributed to the preparation of this manuscript. R. WhiteTraut and K. Norr conceptualized the manuscript and wrote several of the sections. K. Norr took the lead on the framework guiding the study. D. Brandon prepared the section on the Quantitative Data Center, K. Kavanaugh wrote the Qualitative and Mixed Methods Data Center and the Aim 3 mixed methods analysis sections in collaboration with K. Norr, K. Gralton wrote the Implementation Center section. W. Pan wrote the data analysis section, E. Myers wrote the cost analysis section. B. Andrews and M. Msall provided consultation on the infant and parent indicators and provided consultation on the NICU protocol.

Acknowledgements: The authors acknowledge the support of pathways.org for the development of the parent and staff teaching materials.

\section{Authors' information:}

Rosemary White-Traut, PhD, RN, Director, Nursing Research and Evidence-Based Practice, Children's Wisconsin and Professor Emerita, University of Illinois Chicago

Debra Brandon, PhD, RN, Associate Professor, Duke University School of Nursing

Karen Kavanaugh, PhD, RN, Senior Nurse Scientist, Children's Wisconsin and Professor Emerita, University of Illinois Chicago 
Karen Gralton, PhD, RN, Evidence-Based Practice Specialist and Nurse Scientist, Children's Wisconsin

Wei Pan, PhD, Associate Professor, Duke University School of Nursing, and Department of Population Health Sciences, Duke University School of Medicine

Evan R. Myers, MD, MPH, Walter L. Thomas Professor, Division of Women's Community and Population Health, Department of Obstetrics \& Gynecology, Duke University School of Medicine

Bree Andrews, MD, MPH, Associate Professor, Neonatology, University of Chicago, College of Medicine

Michael Msall, MD, Professor, Developmental and Behavioral Pediatrics, University of Chicago, College of Medicine

Kathleen F. Norr, PhD, Senior Scientist, Children's Wisconsin and Professor Emerita, University of Illinois Chicago

\section{References}

1. Walsh EM, Li SX, Black LK, Kuzniewicz M: Incremental Cost of Prematurity by Week of Gestational Age. Am J Perinato/2019, 9(01):e76-e83 DOI: 10.1055/s-0039-1683934.

2. Petrou S, Yiu HH, Kwon J: Economic consequences of preterm birth: a systematic review of the recent literature (2009-2017). Arch Dis Child 2019, 104(5):456-465 DOI: 10.1136/archdischild-2018-315778.

3. Engle WA: Morbidity and mortality in late preterm and early term newborns: A continuum. Clin Perinato/2011, 38(3):493-516 DOI: 10.1016/j.clp.2011.06.009.

4. Gouyan J, lacobelli S, Ferdynus C, Bonsante F: Neonatal problems of late and moderate preterm infants. Sem Fetal Neonat Med 2012, 17(3):146-152 DOI: 10.1016/j.siny.2012.01.015.

5. McCormick MC, Litt JS, Smith VC, Zupancic JA: Prematurity: An overview and public health implications. Annu Rev Public Health 2011, 32:367-379 DOI: 10.1146/annurev-publhealth-090810-182459.

6. Kenney MK, Kogan MD, Toomer S, van Dyck PC: Federal expenditures on maternal and child health in the United States. Matern Child Health J 2012, 16(2):271-287 DOI: 10.1007/s10995-011-0745-5.

7. March of Dimes, Partnership for Maternal, Newborn, and Child Health; Save the Children; World Health Organization: Born too soon: The global action report on preterm birth. Edited by Howson CP, Kinney MV, Lawn JE. Geneva; 2012.

8. Thoyre SM, Shaker CS, Pridham KF: The early feeding skills assessment for preterm infants. Neonatal Netw 2005, 24(3):7-16 DOI: 10.1891/0730-0832.24.3.7.

9. Medoff-Cooper B, Bakewell-Sachs S, Buus-Frank ME, Santa-Donato A, Near-Term Infant Advisory P: The AWHONN Near-Term Infant Initiative: a conceptual framework for optimizing health for near-term infants. J Obstet Gynecol Neonatal Nurs 2005, 34(6):666-671 DOI: $10.1177 / 0884217505281873$.

10. Feldman R, Eidelman Al: Neonatal state organization, neuromaturation, mother-infant interaction, and cognitive development in smallfor-gestational-age premature infants. Pediatrics 2006, 118(3):e869-e878 DOI: 10.1542/peds.2005-2040.

11. White-Traut RC, Nelson MN, Silvestri JM, Cunningham N, Patel M: Responses of preterm infants to unimodal and multimodal sensory intervention. Pediatr Nurs 1997, 23(2):169-175, 193.

12. Nelson MN, White-Traut RC, Vasan U, Silvestri J, Comiskey E, Meleedy-Rey P, Littau S, Gu G, Patel M: One-year outcome of auditorytactile-visual-vestibular intervention in the neonatal intensive care unit: effects of severe prematurity and central nervous system injury. J Child Neurol 2001, 16(7):493-498 DOI: 10.1177/088307380101600706.

13. White-Traut R, Nelson, M., Silvestri, J.M., Ushanalini, V., Patel, M., Cardenas, L.: Feeding readiness behaviors and feeding efficiency in response to ATVV intervention. Newborn Infant Nurs Rev 2002, 2(3):166-173 DOI: https://doi.org/10.1053/nbin.2002.35121.

14. White-Traut RC, Nelson MN, Silvestri JM, Vasan U, Littau S, Meleedy-Rey P, Gu G, Patel M: Effect of auditory, tactile, visual, and vestibular intervention on length of stay, alertness, and feeding progression in preterm infants. Dev Med Child Neurol 2002, 44(2):91-97 DOI: $10.1017 /$ s0012162201001736.

15. Garfield L, Holditch-Davis D, Carter CS, McFarlin BL, Seng JS, Giurgescu C, White-Traut R: A pilot study of oxytocin in low-income women with a low birth-weight infant: Is oxytocin related to posttraumatic stress?Adv Neonatal Care 2019, 19(4):E12-E21 DOI: 10.1097/ANC.0000000000000601.

16. Malin KJ, Johnson TS, McAndrew S, Westerdahl J, Leuthner J, Lagatta J: Infant illness severity and perinatal post-traumatic stress disorder after discharge from the neonatal intensive care unit. Early Hum Dev 2020, 140:104930 DOI:

Page 10/16 
10.1016/j.earlhumdev.2019.104930.

17. Winter L, Colditz PB, Sanders MR, Boyd RN, Pritchard M, Gray PH, Whittingham K, Forrest K, Leeks R, Webb L: Depression, posttraumatic stress and relationship distress in parents of very preterm infants. Arch Women's Ment Health 2018:1-7 DOI: 10.1007/s00737-018-0821-6.

18. Samra HA, Dutcher J, McGrath JM, Foster M, Klein L, Djira G, Hansen J, Wallenburg D: Effect of skin-to-skin holding on stress in mothers of late-preterm infants. Adv Neonatal Care 2015, 15(5):354-364 DOI: 10.1097/ANC.0000000000000223.

19. Treyvaud K, Lee KJ, Doyle LW, Anderson PJ: Very preterm birth influences parental mental health and family outcomes seven years after birth. J Pediatr 2014, 164(3):515-521 DOI: 10.1016/j.jpeds.2013.11.001.

20. Cheng ER, Kotelchuck M, Gerstein ED, Taveras EM, Poehlmann-Tynan J: Postnatal depressive symptoms among mothers and fathers of infants born preterm: Prevalence and impacts on children's early cognitive function. J Dev Behav Pediatr 2016, 37(1):33 DOI: 10.1097/DBP.0000000000000233.

21. Vance AJ, Brandon DH: Delineating among parenting confidence, parenting self-efficacy, and competence. Adv Nursi Sci 2017, 40(4):E18-E37 DOI: 10.1097/ANS.0000000000000179.

22. Ngai F-W, Chan SW-C, Ip W-Y: Predictors and correlates of maternal role competence and satisfaction. Nurs Res 2010, 59(3):185-193 DOI: 10.1097/NNR.0b013e3181dbb9ee.

23. Premji SS, Pana G, Currie G, Dosani A, Reilly S, Young M, Hall M, Williamson T, Lodha AK: Mother's level of confidence in caring for her late preterm infant: A mixed methods study. J Clin Nurs 2018, 27(5-6):e1120-e1133 DOI: 10.1111/jocn.14190.

24. Bozkurt O, Eras Z, Sari FN, Dizdar EA, Uras N, Canpolat FE, Oguz SS: Does maternal psychological distress affect neurodevelopmental outcomes of preterm infants at a gestational age of $\leq 32$ weeks? Early Hum Dev 2017, 104:27-31 DOI: 10.1016/j.earlhumdev.2016.11.006.

25. Baía I, Amorim M, Silva S, Kelly-Irving M, de Freitas C, Alves E: Parenting very preterm infants and stress in neonatal intensive care units. Early Hum Dev 2016, 101:3-9 DOI: 10.1016/j.earlhumdev.2016.04.001.

26. Barradas DT, Wasserman MP, Daniel-Robinson L, Bruce MA, DiSantis KI, Navarro FH, Jones WA, Manzi NM, Smith MW, Goodness BM: Hospital utilization and costs among preterm infants by payer: Nationwide inpatient sample, 2009. Matern Child Health J 2016, 20(4):808-818 DOI: 10.1007/s10995-015-1911-y.

27. March of Dimes: The cost of prematurity to US employers. Edited by March of Dimes Foundation; 2008.

28. Boykova M, Kenner C: Transition from hospital to home for parents of preterm infants. J Perinat Neonatal Nurs 2012, 26(1):81-87 DOI: 10.1097/JPN.0b013e318243e948.

29. Grosse SD, Waitzman NJ, Yang N, Abe K, Barfield WD: Employer-sponsored plan expenditures for infants born preterm. Pediatrics 2017, 140(4):e20171078 DOI: 10.1542/peds.2017-1078.

30. Lipner HS, Huron RF: Developmental and interprofessional care of the preterm infant: Neonatal intensive care unit through high-risk infant follow-up. Pediatric Clinics 2018, 65(1):135-141 DOI: 10.1016/j.pcl.2017.08.026.

31. Liu WF, Laudert S, Perkins B, Macmillan-York E, Martin S, Graven S, Group NQPEE: The development of potentially better practices to support the neurodevelopment of infants in the NICU. J Perinat 2007, 27 Suppl 2:S48-74 DOI: 10.1038/sj.jp.7211844.

32. Guzzetta A, Baldini S, Bancale A, Baroncelli L, Ciucci F, Ghirri P, Putignano E, Sale A, Viegi A, Berardi N et al: Massage accelerates brain development and the maturation of visual function. J Neurosci 2009, 29(18):6042-6051 DOI: 10.1523/jneurosci.5548-08.2009.

33. White-Traut RC, Nelson MN, Burns K, Cunningham N: Environmental influences on the developing premature infant: theoretical issues and applications to practice. J Obstet Gynecol Neonatal Nurs 1994, 23(5):393-401 DOI: 10.1111/j.1552-6909.1994.tb01896.x.

34. Cheng Y-C, Chen L-L, Chang Y-S, Li T-C, Chen C-J, Huang L-C: The effectiveness of learning portfolios in learning participation and learners' perceptions of skills and confidence in the mother of preterm infant. Midwifery 2018, 62:86-91 DOI: 10.1016/j.midw.2018.03.010.

35. Petty J, Jarvis J, Thomas R: Understanding parents' emotional experiences for neonatal education: A narrative, interpretive approach. J Clin Nurs 2019, 28(9-10):1911-1924 DOI: 10.1111/jocn.14807.

36. Petty J, Whiting L, Green J, Fowler C: Parents' views on preparation to care for extremely premature infants at home. Nurs Child Young People 2019, 31(3) DOI: 10.7748/ncyp.2018.e1084.

37. La Monica LH, Blake S, Simmons C, Thompson J, Derouin A: Implementing a parent education program in the Special Care Nursery. J Pediatr Health Care 2019, 33(2):131-137 DOI: 10.1016/j.pedhc.2018.06.007.

38. Carman KL, Dardess P, Maurer M, Sofaer S, Adams K, Bechtel C, Sweeney J: Patient and family engagement: A framework for understanding the elements and developing interventions and policies. Health Aff (Millwood) 2013, 32(2):223-231 DOI:

Page $11 / 16$ 
10.1377/hlthaff.2012.1133.

39. Graffinga G, Barello S, Bonanomi A, Lozza E: Measuring patient engagement: Development and psychometric properties of the Patient Health Engagement (PHE) Scale. Front Psychol 2015, 27(6):274 DOI: 10.3389/fpsyg.2015.00274.

40. De Bernardo G, Svelto M, Giordano M, Sordino D, Riccitelli M: Supporting parents in taking care of their infants admitted to a neonatal intensive care unit: A prospective cohort pilot study. Ital J Pediatr 2017, 43(1):36 DOI: 10.1186/s13052-017-0352-1.

41. Castel S, Creveuil C, Beunard A, Blaizot X, Proia N, Guillois B: Effects of an intervention program on maternal and paternal parenting stress after preterm birth: A randomized trial. Early Hum Dev 2016, 103:17-25 DOI: 10.1016/j.earlhumdev.2016.05.007.

42. White-Traut R, Norr KF, Fabiyi C, Rankin KM, Li Z, Liu L: Mother-infant interaction improves with a developmental intervention for mother-preterm infant dyads. Infant Behav Dev 2013, 36(4):694-706 DOI: https://doi.org/10.1016/j.infbeh.2013.07.004.

43. Kaaresen PI, Ronning JA, Ulvund SE, Dahl LB: A randomized, controlled trial of the effectiveness of an early-intervention program in reducing parenting stress after preterm birth. Pediatrics 2006, 118(1):E9-19 DOI: 10.1542/peds.2005-1491.

44. Msall M: Optimizing parental involvement in caring for preterm infants. J Perinatol 2015, 35(9):669-671 DOI: 10.1038/jp.2015.60.

45. Samra HA, McGrath JM, Fischer S, Schumacher B, Dutcher J, Hansen J: The NICU parent risk evaluation and engagement model and instrument (PREEMI) for neonates in intensive care units. J Obstet Gynecol Neonatal Nurs 2015, 44(1):114-126 DOI: 10.1111/15526909.12535.

46. Ardal F, Sulman J, Fuller-Thomson E: Support like a walking stick: Parent-buddy matching for language and culture in the NICU. Neonatal Netw 2011, 30(2):89-98 DOI: 10.1891/0730-0832.30.2.89.

47. Umberger E, Canvasser J, Hall SL: Enhancing NICU parent engagement and empowerment. Semin Pediatr Surg 2018, 27(1): 19-24. DOI: https://doi.org/10.1053/j.sempedsurg.2017.11.004

48. White-Traut RC, Rankin KM, Yoder JC, Liu L, Vasa R, Geraldo V, Norr KF: Influence of H-HOPE intervention for premature infants on growth, feeding progression and length of stay during initial hospitalization. J Perinatol 2015, 35(8):636-641 DOI: 10.1038/jp.2015.11.

49. Medoff-Cooper B, Rankin K, Li Z, Liu L, White-Traut R: Multi-sensory intervention for preterm infants improves sucking organization. Adv Neonatal Care 2015, 15(2):142 DOI: 10.1097/ANC.0000000000000166.

50. White-Traut RC, Nelson MN: Maternally administered tactile, auditory, visual, and vestibular stimulation: Relationship to later interactions between mothers and premature infants. Res Nurs Health 1988, 11(1):31-39 DOI: 10.1002/nur.4770110106.

51. White-Traut R, Studer T, Meleedy-Rey P, Murray P, Labovsky S, Kahn J: Pulse rate and behavioral state correlates after auditory, tactile, visual, and vestibular intervention in drug-exposed neonates. J Perinatol 2002, 22(4):291-299 DOI: 10.1038/sj.jp.7210695.

52. White-Traut RC, Nelson MN, Silvestri JM, Patel M, Vasan U, Han BK, Cunningham N, Burns K, Kopischke K, Bradford L: Developmental intervention for preterm infants diagnosed with periventricular leukomalacia. Res Nurs Health 1999, 22(2):131-143 DOI:

10.1002/(sici)1098-240x(199904)22:2<131::aid-nur5>3.0.co;2-e.

53. White-Traut RC, Nelson MN, Silvestri JM, Patel MK, Kilgallon D: Patterns of physiologic and behavioral response of intermediate care preterm infants to intervention. Pediatr Nurs 1993, 19(6):625-629.

54. White-Traut RC, Tubeszewski KA: Multimodal stimulation of the premature infant. J Pediatr Nurs 1986, 1(2):90-95.

55. White-Traut R, Rankin KM, Pham T, Li Z, Liu L: Preterm infants' orally directed behaviors and behavioral state responses to the integrated H-HOPE intervention. Infant Behav Dev 2014, 37(4):583-596 DOI: https://doi.org/10.1016/j.infbeh.2014.08.001.

56. Vonderheid S, Rankin K, Norr K, Vasa R, Hill S, White-Traut R: Health care use outcomes of an integrated hospital-to-home motherpreterm infant intervention. J Obstet Gynecol Neonatal Nurs 2016, 45(5):625-638 DOI: 10.1016/j.jogn.2016.05.007.

57. Vonderheid S, Park C, Rankin K, Norr K, White-Traut R: Impact of an integrated mother-preterm infant intervention on birth hospitalization costs. J Perinato/ 2020:1-9 DOI: 10.1038/s41372-019-0567-7.

58. Damschroder LJ, Aron DC, Keith RE, Kirsh SR, Alexander JA, Lowery JC: Fostering implementation of health services research findings into practice: A consolidated framework for advancing implementation science. Implemen Sci 2009, 4(1):50 DOI: 10.1186/1748-59084-50.

59. Ilott I, Gerrish K, Booth A, Field B: Testing the Consolidated Framework for Implementation Research on health care innovations from South Yorkshire. J Eval Clin Pract 2013, 19(5):915-924 DOI: 10.1111/j.1365-2753.2012.01876.x.

60. Powell BJ, Proctor EK, Glass JE: A systematic review of strategies for implementing empirically supported mental health interventions. Res Soc Work Pract 2014, 24(2):192-212 DOI: 10.1177/1049731513505778.

61. Kirk MA, Kelley C, Yankey N, Birken SA, Abadie B, Damschroder L: A systematic review of the use of Consolidated Framework for Implementation Research. Implemen Sci 2016, 11(72):1-13 DOI: 10.1186/s13012-016-0437-z. 
62. Balas MC, Burke WJ, Gannon D, Cohen MZ, Colburn L, Bevil C, Franz D, Olsen KM, Ely EW, Vasilevskis EE: Implementing the awakening and breathing coordination, delirium monitoring/management, and early exercise/mobility bundle into everyday care: Opportunities, challenges, and lessons learned for implementing the ICU Pain, Agitation, and Delirium Guidelines. Crit Care Med 2013, 41(9 Suppl 1):S116-127 DOI: 10.1097/CCM.0b013e3182a17064.

63. Liang S, Kegler MC, Cotter M, Phillips E, Beasley D, Hermstad A, Morton R, Martinez J, Riehman K: Integrating evidence-based practices for increasing cancer screenings in safety net health systems: A multiple case study using the Consolidated Framework for Implementation Research. Implemen Sci 2015, 11(1):109 DOI: 10.1186/s13012-016-0477-4.

64. Sopcak N, Aguilar C, O’Brien MA, Nykiforuk C, Aubrey-Bassler K, Cullen R, Grunfeld E, Manca DP: Implementation of the BETTER 2 program: A qualitative study exploring barriers and facilitators of a novel way to improve chronic disease prevention and screening in primary care. Implemen Sci 2016, 11(1):158 DOI: 10.1186/s13012-016-0525-0.

65. Garbutt JM, Dodd S, Walling E, Lee AA, Kulka K, Lobb R: Barriers and facilitators to HPV vaccination in primary care practices: A mixed methods study using the Consolidated Framework for Implementation Research. BMC Fam Pract 2018, 19(1):53 DOI: $10.1186 / \mathrm{s} 12875-018-0750-5$.

66. Ware P, Ross HJ, Cafazzo JA, Laporte A, Gordon K, Seto E: Evaluating the implementation of a mobile phone-based telemonitoring program: Longitudinal study guided by the Consolidated Framework for Implementation Research. J Med Internet Res 2018, 6(7) DOI: $10.2196 / 10768$.

67. Warner G, Lawson B, Sampalli T, Burge F, Gibson R, Wood S: Applying the consolidated framework for implementation research to identify barriers affecting implementation of an online frailty tool into primary health care: A qualitative study. BMC Health Serv Res 2018, 18(1):395 DOI: 10.1186/s12913-018-3163-1.

68. White M, Randall K, Capo-Chichi N, Sodogas F, Quenum S, Wright K, Close K, Russ S, Sevdalis N, Leather A: Implementation and evaluation of nationwide scale-up of the Surgical Safety Checklist. Br J Surg 2019, 106(2):e91-e102 DOI: 10.1002/bjs.11034.

69. Barnes-Daly MA, Pun BT, Harmon LA, Byrum DG, Kumar VK, Devlin JW, Stollings JL, Puntillo KA, Engel HJ, Posa PJ: Improving health care for critically ill patients using an evidence-based collaborative approach to ABCDEF bundle dissemination and implementation. Worldviews Evid Based Nurs 2018, 15(3):206-216 DOI: 10.1111/wvn.12290.

70. Crowley K, Mishra A, Cruz-Cano R, Gold R, Kleinman D, Agarwal R: Electronic health record implementation findings at a large, suburban health and human services department. J Public Health Manag Pract 2019, 25(1):E11-E16 DOI: 10.1097/PHH.0000000000000768.

71. Proctor E, Silmere H, Raghavan R, Hovmand P, Aarons G, Bunger A, Griffey R, Hensley M: Outcomes for implementation research: conceptual distinctions, measurement challenges, and research agenda. Admin Pol Ment Health 2011, 38(2):65-76 DOI: 10.1007/s10488-010-0319-7.

72. Shelton R, Lee ML: Sustaining evidence-based interventions and policies: Recent innovations and future directions in implementation science. AJPH 2019, 109(52) DOI:

73. Castro FG, Barrera Jr. M, Martinez Jr. CR: The cultural adaptation of prevention interventions: Resolving tensions between fidelity and fit. Preven Sci 2004, 5(1):41-45 DOI: 10.1023/b:prev.0000013980.12412.cd.

74. Abry T, Hulleman CS, Rimm-Kaufman SE: Using indices of fidelity to intervention core components to identify program active ingredients. Am J Eval 2014, 36(3):320-338 DOI: https://doi.org/10.1177/1098214014557009.

75. Curran GM, Bauer M, Mittman B, Pyne JM, Stetler C: Effectiveness-implementation hybrid designs: Combining elements of clinical effectiveness and implementation research to enhance public health impact. Med Care 2012, 50(3):217 DOI: 10.1097/MLR.0b013e3182408812.

76. Hemming K, Lilford R, Girling AJ: Stepped-wedge cluster randomised controlled trials: A generic framework including parallel and multiple-level designs. Stat Med 2015, 34(2):181-196 DOI: 10.1002/sim.6325.

77. Barker D, McElduff P, D’Este C, Campbell MJ: Stepped wedge cluster randomised trials: A review of the statistical methodology used and available. BMC Med Res Methodol 2016, 16:69 DOI: 10.1186/s12874-016-0176-5.

78. Hemming K, Haines T, Chilton P, Girling A, Lilford R: The stepped wedge cluster randomised trial: Rationale, design, analysis, and reporting. The BMJ 2015, 350:h391 DOI: 10.1136/bmj.h391.

79. Hussey MA, Hughes JP: Design and analysis of stepped wedge cluster randomized trials. Contemp Clin Trials 2007, 28(2):182-191 DOI: $10.1016 /$ j.cct.2006.05.007.

80. Damschroder LJ, Goodrich DE, Robinson CH, Fletcher CE, Lowery JC: A systematic exploration of differences in contextual factors related to implementing the MOVE! weight management program in VA: A mixed methods study. BMC Health Serv Res 2011, 11(1):248 DOI: 10.1186/1472-6963-11-248. 
81. Damschroder LJ, Lowery JC: Evaluation of a large-scale weight management program using the consolidated framework for implementation research (CFIR). Implemen Sci 2013, 8(1):51 DOI: 10.1186/1748-5908-8-51.

82. Martin JA, Hamilton BE, Osterman MJK, Driscoll AK, Drake P: Births: Final data for 2016. In: Nat/ Vital Stat Rep. vol. 67. Hyattsville, MD: National Center for Health Statistics; 2018.

83. Hemming K, Girling A: A menu-driven facility for power and detectable-difference calculations in stepped-wedge cluster-randomized trials. Stata 2014, 14(2):363-380 DOI: https://doi.org/10.1177/1536867X1401400208.

84. Thompson DM, Fernald DH, Mold JW: Intraclass correlation coefficients typical of cluster-randomized studies: estimates from the Robert Wood Johnson Prescription for Health projects. Ann Fam Med 2012, 10(3):235-240 DOI: 10.1370/afm.1347.

85. Shackleton N, Hale D, Bonell C, Viner R: Intraclass correlation values for adolescent health outcomes in secondary schools in 21 European countries. SSM Popul Health 2016, 2:217-225 DOI: 10.1016/j.ssmph.2016.03.005.

86. Gomes M, Ng ES-W, Grieve R, Nixon R, Carpenter J, Thompson SG: Developing appropriate methods for cost-effectiveness analysis of cluster randomized trials. Med Decis Making 2012, 32(2):350-361 DOI: 10.1177/0272989X11418372.

87. Janjua NZ, Khan MI, Clemens JD: Estimates of intraclass correlation coefficient and design effect for surveys and cluster randomized trials on injection use in Pakistan and developing countries. Trop Med Int Health 2006, 11(12):1832-1840 DOI: 10.1111/j.13653156.2006.01736.x.

88. Kul S, Vanhaecht K, Panella M: Intraclass correlation coefficients for cluster randomized trials in care pathways and usual care: Hospital treatment for heart failure. BMC Health Serv Res 2014, 14(1):84 DOI: 10.1186/1472-6963-14-84.

89. White-Traut RC, Berbaum ML, Lessen B, McFarlin B, Cardenas L: Feeding readiness in preterm infants: The relationship between preterm behavioral state and feeding readiness behaviors and efficiency during transition from gavage to oral feeding. $M C N$ : $A m J$ Matern Child Nurs 2005, 30(1):52-59.

90. White-Traut RC, Pate CM: Modulating infant state in premature infants. J Pediatr Nurs 1987, 2(2):96-101.

91. White-Traut RC, Goldman MBC: Premature infant massage: Is it safe? Pediatr Nurs 1988, 14(4):285-289.

92. Walton H, Spector A, Tombor I, Michie S: Measures of fidelity of delivery of, and engagement with, complex, face-to-face health behaviour change interventions: A systematic review of measure quality. Br J Health Psychol 2017, 22(4):872-903 DOI: $10.1111 /$ bjhp. 12260 .

93. Toomey E, Matthews J, Hurley DA: Using mixed methods to assess fidelity of delivery and its influencing factors in a complex selfmanagement intervention for people with osteoarthritis and low back pain. BMJ Open 2017, 7(8):e015452 DOI: 10.1136/bmjopen2016-015452.

94. Wandersman A, Chien VH, Katz J: Toward an evidence-based system for innovation support for implementing innovations with quality: Tools, training, technical assistance, and quality assurance/quality improvement. Am J Community Psychol2012, 50(34):445-459 DOI: 10.1007/s10464-012-9509-7.

95. Brazy JE, Eckerman CO, Oehler JM, Goldstein RF, Angela M: Nursery neurobiologic risk score: Important factors in predicting outcome in very low birth weight infants. J Pediatr 1991, 118(5):783-792 DOI: https://doi.org/10.1016/S0022-3476(05)80047-2.

96. Brazy JE, Goldstein RF, Oehler JM, Gustafson KE, Thompson RJ: Nursery neurobiologic risk score: Levels of risk and relationships with nonmedical factors. J Dev Behav Pediatr 1993 DOI: https://doi.org/10.1097/00004703-199312010-00003.

97. Pan W, Bai H: Propensity score analysis: Fundamentals and developments. Guilford Publications; 2015 Apr 7.

98. Pan W, Bai H: Propensity score methods in nursing research: Take advantage of them but proceed with caution. Nurs Res 2016, 65(6):421-424 DOI: 10.1097/NNR.0000000000000189.

99. Rosenbaum PR, Rubin DB: The central role of the propensity score in observational studies for causal effects. Biometrika 1983, 70(1):41-55 DOI: https://doi.org/10.1093/biomet/70.1.41.

100. Rosenbaum PR, Rubin DB: Constructing a control group using multivariate matched sampling methods that incorporate the propensity score. Am Stat 1985, 39(1):33-38 DOI: 10.1080/00031305.1985.10479383.

101. Hughes JP, Granston TS, Heagerty PJ: Current issues in the design and analysis of stepped wedge trials. Contemp Clin Trials 2015, 45:55-60 DOI: 10.1016/j.cct.2015.07.006. .

102. Ramsey SD, Willke RJ, Glick H, Reed SD, Augustovski F, Jonsson B, Briggs A, Sullivan SD: Cost-effectiveness analysis alongside clinical trials II-an ISPOR Good Research Practices Task Force report. Value Health 2015, 18(2):161-172 DOI: 10.1016/j.jval.2015.02.001.

103. Briggs AH, Weinstein MC, Fenwick EA, Karnon J, Sculpher MJ, Paltiel AD: Model parameter estimation and uncertainty analysis: a report of the ISPOR-SMDM Modeling Good Research Practices Task Force Working Group-6. Med Decis Making 2012, 32(5):722-732

Page $14 / 16$ 
DOI: $10.1177 / 0272989 \times 12458348$.

104. Creswell JW, Plano Clark VL: Designing and conducting mixed methods research, 3rd edn. Thousand Oaks, CA: Sage Publications; 2018.

105. Hsieh HF, Shannon SE: Three approaches to qualitative content analysis. Qual Health Res 2005, 15(9):1277-1288 DOI: $10.1177 / 1049732305276687$.

106. Miles MS, Holditch-Davis D, Schwartz TA, Scher M: Depressive symptoms in mothers of prematurely born infants. J Dev Behav Pediatr 2007, 28(1):36-44 DOI: 10.1097/01.DBP.0000257517.52459.7a.

107. Črnčec R, Barnett B, Matthey S: Karitane Parenting Confidence Scale: Manual. Sydney: Sydney South West Area Health Service 2008.

108. Pilkonis PA, Choi SW, Reise SP, Stover AM, Riley WT, Cella D, Group PC: Item banks for measuring emotional distress from the PatientReported Outcomes Measurement Information System (PROMIS®): Depression, anxiety, and anger. Assessment 2011, 18(3):263-283 DOI: $10.1177 / 1073191111411667$.

\section{Tables}

Table 1. Expected H-HOPE Cohort and Site Characteristics

\begin{tabular}{|c|c|c|c|c|c|c|c|c|c|c|c|c|}
\hline \multirow{2}{*}{$\begin{array}{l}\text { NICU } \\
\text { Site }^{1}\end{array}$} & \multirow{2}{*}{$\begin{array}{l}\text { NICU } \\
\text { Level }\end{array}$} & \multirow{2}{*}{$\begin{array}{l}\text { Annual } \\
\text { Admiss }^{2}\end{array}$} & \multirow{2}{*}{$\begin{array}{l}\text { \# } \\
\text { Eligible } \\
\text { in } \\
6 \\
\text { Months }^{3}\end{array}$} & \multirow{2}{*}{$\begin{array}{l}\# \\
\text { Enrolled }^{3}\end{array}$} & \multirow{2}{*}{$\begin{array}{l}\# \\
\text { Retained }^{3}\end{array}$} & \multicolumn{5}{|c|}{ Race/ethnicity ${ }^{4} \%$} & \multirow{2}{*}{$\begin{array}{l}\% \\
\text { Medicaid }\end{array}$} & \multirow[t]{2}{*}{ Location } \\
\hline & & & & & & Wh & Blk & $\begin{array}{l}\text { Am } \\
\text { Ind }\end{array}$ & Asian & Latinx & & \\
\hline Site 1 & III/IV & 184 & 62 & 44 & 31 & 16 & 71.2 & 0 & 0 & 12.8 & 74.5 & Urban \\
\hline \multirow{2}{*}{$\begin{array}{l}\text { Sites } 2 \\
\text { and } 3\end{array}$} & \multirow{2}{*}{$\begin{array}{l}\text { III/IV, } \\
\text { II }\end{array}$} & 454 & 222 & 155 & 108 & 38 & 48 & 8 & 2 & 4 & 70 & Rural/ \\
\hline & & 119 & 30 & 21 & 15 & & & & & & & Suburban \\
\hline Site 4 & III & 297 & 148 & 111 & 77 & 62.8 & 6 & 0 & 15.5 & 14.9 & 40 & Suburban \\
\hline Site 5 & III & 150 & 69 & 48 & 34 & 89 & 1.8 & 3 & 2.2 & 4 & 36 & Rural \\
\hline TOTAL(s) & & 1205 & 542 & 379 & 265 & 42.7 & 34.9 & 4.5 & 4.8 & 7 & & \\
\hline
\end{tabular}

${ }^{1}$ In order of Random Assignment; ${ }^{2}$ Annual Admissions for $23-35$ wk. GA; ${ }^{3}$ \# eligible, \# enrolled is at a $70 \%$ enrollment rate \& \# retained at 6-weeks post discharge for H-HOPE Cohort; ${ }^{4}$ White, Black/African American, American Indian, Latinx 


\begin{tabular}{|c|c|c|c|c|c|}
\hline \multirow{2}{*}{$\begin{array}{l}\text { Outcome } \\
\text { Variable }\end{array}$} & \multirow[t]{2}{*}{ Operational Measure } & \multicolumn{3}{|c|}{ Timing } & \multirow{2}{*}{$\begin{array}{l}\text { Data } \\
\text { Source }\end{array}$} \\
\hline & & $\mathrm{D} / \mathrm{C}$ & $\begin{array}{l}2- \\
\text { wks }\end{array}$ & $\begin{array}{l}\text { 6- } \\
\text { wks }\end{array}$ & \\
\hline Infant & 1. Growth-weight and length; & - & $\bullet$ & - & EHR \\
\hline (Primary) & 2. Fewer acute care visits discharge to 6 -weeks post discharge & & & & $\begin{array}{l}\text { Parent } \\
\text { report }\end{array}$ \\
\hline $\begin{array}{l}\text { Costs } \\
\text { (Secondary) }\end{array}$ & $\begin{array}{l}\text { 3. Average hospitalization cost/day (direct costs) from eligibility for H-HOPE through } \\
\text { discharge, adjusted for the H-HOPE cohort only by adding the cost per infant to } \\
\text { implement H-HOPE (training, coordinating, time to deliver H-HOPE and to arrange } \\
\text { screen time visits) }\end{array}$ & $\bullet$ & & & $\begin{array}{l}\text { Hospital } \\
\text { cost } \\
\text { data \& } \\
\text { H-HOPE } \\
\text { records }\end{array}$ \\
\hline \multirow{5}{*}{$\begin{array}{l}\text { Parent } \\
\text { (Exploratory) }\end{array}$} & 4. \# Parent NICU visits/days of hospitalization (each parent) & $\bullet$ & & $\bullet$ & EHR \\
\hline & 5. Parental Worry [106] & $\bullet$ & & & \multirow{4}{*}{$\begin{array}{l}\text { Parent } \\
\text { Survey }\end{array}$} \\
\hline & 6. Karitane Parenting Confidence Scale [107] & $\bullet$ & & & \\
\hline & 7. Parent depressive symptoms, PCORI short form [108] & & & & \\
\hline & 8, Parent anxiety PCORI short form [108] & & & & \\
\hline
\end{tabular}

\section{Figures}

\begin{tabular}{|c|c|c|c|c|c|c|c|}
\hline \multirow[b]{2}{*}{ NICU Site } & \multicolumn{7}{|c|}{ Step } \\
\hline & 0 & 1 & 2 & 3 & 4 & 5 & 6 \\
\hline 1 & Pre-I \& P & $E$ & $\mathrm{R}$ & $\mathrm{s}$ & & & \\
\hline 2 and 3 & & Pre-I \& P & $E$ & $R$ & $\mathrm{~S}$ & & \\
\hline 4 & & & Pre-I \& P & $E$ & $\mathrm{R}$ & $\mathrm{S}$ & \\
\hline 5 & & & & Pre-I \& P & $\mathrm{E}$ & $\mathrm{R}$ & $s$ \\
\hline
\end{tabular}

\section{Figure 1}

Incomplete Stepped Wedge Design. Pre-I \& P = 6 months of pre-implementation plus Planning and Engaging during which Pre-H-HOPE Comparison Cohort Data are collected simultaneously, $E=6$ months of Executing at the end of which posttest will be taken, $R=2$ months of Reflecting and Evaluating, $S=6$ months of Sustaining. The order of implementation was randomly assigned. 